Revue d'histoire de l'Amérique française

REVUE D.HISTOIRE DE L'AMÉRIQUE FRANÇAISE

\title{
Le nationalisme et les partis politiques
}

\section{André-J. Bélanger et Vincent Lemieux}

Volume 22, numéro 4, mars 1969

URI : https://id.erudit.org/iderudit/302826ar

DOI : https://doi.org/10.7202/302826ar

Aller au sommaire du numéro

Éditeur(s)

Institut d'histoire de l'Amérique française

ISSN

0035-2357 (imprimé)

1492-1383 (numérique)

Découvrir la revue

Citer cet article

Bélanger, A.-J. \& Lemieux, V. (1969). Le nationalisme et les partis politiques. Revue d'histoire de l'Amérique française, 22(4), 539-563.

https://doi.org/10.7202/302826ar d'utilisation que vous pouvez consulter en ligne.

https://apropos.erudit.org/fr/usagers/politique-dutilisation/ 
André-J. Bélanger

et

Vincent Lemieux

\section{LE NATIONALISME ET LES PARTIS POLITIQUES}

Marc La Terreur

Commentaire 


\section{André-J. Bélanger}

Maître ès arts en Science politique de l'Université McGill. Etudes supérieures en Science politique à l'Université de Paris.

Professeur auxiliaire au Département de Science politique de l'Université Laval (Québec).

Chargé d'un cours sur les idéologies politiques au Québec.

\section{Vincent Lemieux}

Maître en Science politique de l'Université Laval (Québec). Diplômé d'études supérieures en recherches politiques, Université de Paris. Chercheur spécialisé en analyse et théories politiques.

Directeur du Département de Science politique de l'Université Laval (Québec).

A publié de nombreux articles dans la Revue canadienne de Science politique et dans Recherches sociographiques.

Ouvrage en préparation: Provincial Party Politics in Canada (Editions Prentice Hall).

\section{Marc La Terreur}

Licencié ès Lettres (histoire) de l'Université Laval (Québec). Recherches orientées surtout en histoire politique.

Professeur d'histoire du Canada à l'Université Laval (Québec).

A collaboré à plusieurs publications notamment:

Regionalism in the Canadian Community 1867-1967 (sous presse, aux Editions University of Toronto Press);

Rapport sur Le Parti conservateur et le Québec 1930-1965, dans le cadre de la Commission royale d'enquête sur le bilinguisme et le biculturalisme. 


\section{LE NATIONALISME ET LES PARTIS POLITIQUES}

Le sujet proposé permet de poser deux questions qui correspondent à deux développements distincts. Ainsi dans un premier temps nous nous demanderons quelle place le nationalisme a faite aux formations politiques et au politique en général. Dans un second temps nous nous interrogerons sur l'attitude des partis politiques vis-à-vis du nationalisme.

\section{I}

Avant d'aborder tout de go la première question, quelques distinctions s'imposent. Le nationalisme est susceptible d'être perçu comme une idéologie globale, totale, c'est-à-dire une idéologie qui se suffit à elle-même et qui évolue en demeurant sensiblement fidèle à elle-même. On entend encore parler des "nationalistes" comme formant un groupe homogène, constitué et continu dans le temps. Depuis quelques années, la multiplicité des tendances nationalistes s'est suffisamment imposée pour dégager une impression sinon d'incohérence, du moins de tiraillements, et parfois même de division profonde et d'opposition. Néanmoins, certains persistent à croire que la diversité des "nationalismes" ou, si on préfère, la diversité des formes que peut prendre le nationalisme ne s'est fait jour que depuis ces dernières années. Sans reculer jusqu'au siècle dernier, où alors de toute évidence le nationalisme de Papineau se distinguait à bien des égards de celui qui devait s'imposer plusieurs décennies plus tard, il est manifeste que le nationalisme s'est trouvé le ressort d'une multitude d'idéologies qui n'avaient souvent en commun que leur profond attachement à la "nation". Nous pensons plus particulièrement à la période des années '30 qui a progressivement révélé combien le nationalisme était capable de ramification et de division. Les penseurs officiels de l'Action nationale de l'époque se verront souvent dépassés et débordés 
par des jeunes et des moins jeunes formés à des écoles parfois absolument étrangères au nationalisme comme tel. La filière d'André Laurendeau est particulièrement significative à cet égard: nourri dès son enfance aux sources mêmes du nationalisme le plus farouchement conservateur, il s'en dégagera pour lui préférer un nationalisme ouvert au personnalisme de Mounier. L'évolution du périodique La Relève est tout aussi conforme à ces changements que dut subir une pensée nationaliste somme toute assez figée à ses débuts.

Il faut donc, en parlant de nationalisme, préciser de façon satisfaisante de quel type de nationalisme il s'agit et, forcément, à quelle époque il s'est imposé. Nous fondant sur les sujets proposés dans le cadre du présent colloque, nous avons cru désirable de nous arrêter à la forme de nationalisme la plus familière, la plus répandue à une certaine époque, celle qui s'est reflétée chez le chanoine Groulx et son entourage, de même que chez plusieurs collaborateurs de l'Action nationale du début des années trente. Nous sommes très conscients de ne pas choisir la plus dynamique, ni celle qui a le moins prêté flanc à la critique, au quolibet ou à la caricature. Nous portons notre choix sur celle-ci, parce qu'à travers le temps, malgré certaines transformations qu'elle a dû subir, elle est demeurée un bloc passablement cohérent susceptible de présenter les avantages d'un type idéal. Or c'est précisément à ce titre que nous désirons l'employer pour mieux faire saisir la distance qui a séparé cette idéologie de la chose publique. Bien sûr, la réalité comporte des bavures, des incohérences que le type idéal ne peut retenir.

Quelle place donc le nationalisme officiel a-t-il réservée aux formations politiques et au politique pris dans son sens plus général ? On peut dire que dans l'ensemble les nationalistes se sont renfrognés assez longtemps dans une attitude de mépris et de refus. Attitude de mépris à l'égard des partis politiques dont ils ont constamment condamné les compromissions, la lâcheté, la corruption. Il s'agit bien là d'une constante du nationalisme: les formations politiques sont constituées de traîtres, de traîtres à la "race", de traîtres à la "nation". Ces politiciens ont pour 
fonction secondaire de distraire la "masse" de ses préoccupations nationales en créant et maintenant ce que les nationalistes appelleront: "l'esprit de parti" et le "régime des partis".

Cette distance que la pensée nationaliste prend à l'endroit du personnel politique répond à une logique assez simple. L'exercice malhonnête de la politique sera intimement lié à l'appartenance de ces agents à une classe sociale et plus précisément à une fonction dans la société. La condamnation des "politiciens" est étroitement associée à une condamnation tout aussi radicale d'une certaine bourgeoisie, de cette bourgeoisie qui s'est profondément compromise aux intérêts étrangers et plus souvent qu'autrement, opposés au "national". Le chanoine Groulx a été très explicite à ce propos :

La bourgeoisie a fait de la politique son affaire (...), Mais, son industrie; elle y a laissé son âme ${ }^{1}$.

la bourgeoisie politicienne ne s'est pas arrêtée là. Après avoir identifié le politique et le national, elle a évincé, expulsé le national du politique ${ }^{2}$.

Ceci a eu pour conséquence, que

entraîné, guidé par sa bourgeoisie, le groupe ethnique au Canada qui a le plus farouchement pratiqué la discipline de parti, la fidélité absolue, superstitieuse au parti, c'est le nôtre ${ }^{3}$.

Primo, condamnation d'une classe qui a renié son identité collective; et secundo, condamnation d'une classe qui a entraîné la collectivité à sa suite en l'embrigadant et en l'enfermant dans le système des partis. Enfin, les formations politiques seront bannies pour avoir partie liée avec "la dictature financière". Elles sont des représentantes des trusts, des monopoles, elles sont à la solde du monde de la finance et de l'argent en général.

Pour ces raisons, la pensée nationaliste sera comme paralysée lorsque confrontée aux partis. S'adressant à des étudiants

${ }^{1}$ L. Groulx, "La bourgeoisie et le national" in L'Avenir de notre bourgeoisie (Montréal, Valiquette, 1939), 107.

2 Ibid., 110.

3 Ibid., 109. 
en 1941, le chanoine Groulx leur prescrivait d'opérer une "réforme de la conscience collective" et une "substitution de l'esprit national à l'esprit d'oligarchie ou de clan" ${ }^{4}$ avant de se lancer dans l'arène politique. Et, ne se faisant à l'époque aucune illusion sur son emprise auprès de la société dans son ensemble, il ajoutait qu'un des torts des partis de réforme dans le passé avait été "d'aller demander un suffrage national à un électorat qui n'avait rien de national" " 5 . Au fond, les partis seront surtout honnis pour leur ascendant auprès des électeurs. La position des nationalistes vis-à-vis des partis politiques en sera toujours une de faiblesse profondément ressentie. Le déséquilibre des forces poussera le nationalisme à se recroqueviller et à entretenir une aigreur bien nourrie envers les partis toujours vainqueurs. Il faut ajouter que l'expérience de l'Action libérale nationale ne sera pas susceptible d'assouplir ce raidissement.

Donc, mépris des hommes politiques, condamnation d'une certaine bourgeoisie. Encore faut-il voir que le fossé est bien plus profond qu'il ne le paraît à première vue. Car en plus d'être diamétralement opposées, ces forces sont irréductibles aux yeux de ces nationalistes.

L'idéologie nationaliste de type conservateur dont il est question s'est trouvée privée en quelque sorte de la composante politique. Cette droite nostalgique a pu sans trop de difficultés se constituer une structure sociale qui renvoyait peut-être à une époque révolue. Néanmoins elle est parvenue à restituer un univers économique et social propre à une société traditionnelle. Est-il nécessaire de rappeler ces caractéristiques que maints articles ont reprises: la famille fondement de la société, le ruralisme garant de nos traditions, la langue gardienne de la foi et, réciproquement, la foi gardienne de la langue, etc. L'aspect économique est plus intéressant à retenir à ce stade-ci. Car, à ce point de vue, le nationalisme officiel, quoiqu'un peu embarrassé, s'est tout de même construit un univers conforme au passé, même 1941), 48.

${ }^{4} \mathrm{~L}$. Groulx, Paroles à des étudiants (L'Action nationale, Montréal, 5 Ibid., 48. 
si celui-ci offrait peu de correspondance avec la situation présente. L'achat chez nous, le retour à la terre, la colonisation et, finalement, le corporatisme, se présenteront comme des éléments de réponse. À remarquer, au passage, que les partis politiques puiseront peu dans cette idéologie qui, en termes de réponse à la dépression, leur paraîtra offrir des solutions dérisoires. En opposition au libéralisme économique et à la révolution industrielle, le nationalisme aura donc au moins quelques satisfactions: celles entre autres de croire qu'au départ la "mission" du Canada français n'est pas d'ordre économique, celle de croire également qu'un retour au statu quo ante présente des garanties de récupération nationale. Bref, le passé, "notre maître", offrait sur ce plan des solutions.

$\mathrm{Au}$ point de vue politique, le problème s'est posé bien autrement. Le nationalisme traditionnel s'est trouvé encore plus dépourvu devant le politique que devant l'économique. Le passé n'offrait aucun modèle de référence. Ainsi, les nationalistes ont perçu beaucoup plus clairement les valeurs qu'ils rejetaient que celles qu'ils consentaient à faire leurs.

Le nationalisme a exécuté assez sommairement le libéralisme politique. Pour le chanoine Groulx, le libéralisme dérivait en gros de la Réforme; le compromis entre sa pensée et l'idéologie devenait impossible puisque ces deux univers se présentaient à ses yeux comme foncièrement incompatibles. Le chanoine a établi clairement cette distinction:

L'on sait comme en certaines provinces de la Puissance, l'esprit de la Réforme a faussé les notions de la justice, du droit, de l'Etat et de son autorité, les relations des classes, du capital et du travail; l'on sait comme on y sacrifie facilement à l'Etat des droits naturels du père de famille sur l'éducation de ses enfants... comme on y entend mal les rapports du temporel et du spirituel dans la vie publique ${ }^{6}$.

Nul ne me persuadera qu'un peuple de deux à trois millions de catholiques existe au Canada pour dire 33.

6 Lionel Groulx, Orientations (Editions du Zodiaque, Montréal, 1935), 
la même chose que les autres, se contenter de la même presse, du même théâtre, de la même législation sociale, de la même philosophie de la vie, quand ces autres sont des chrétiens séparés de la vraie foi par le grand malheur de la Réforme ?

Les institutions libérales faisaient partie de l'arsenal du conquérant et colonisateur, elles relevaient d'une logique qui lui était étrangère. Elles étaient animées soit par des anglophones, soit par des "politiciens"; dans les deux cas, il s'agissait d'antinationaux. Le libéralisme politique tel qu'il se pratiquait au pays excluait systématiquement la dimension nationale.

La démocratie a toujours été perçue par le milieu nationaliste des années trente comme un élément étranger à la société québecoise, un élément que des agents extérieurs avaient imposé. Bourassa ne sera jamais un chaud partisan du suffrage universel. Son opposition au vote de la femme est désormais célèbre. Ses successeurs se montreront encore plus réservés sinon hostiles au vote populaire. Le chanoine Groulx, à de multiples occasions, manifestera son antipathie pour un système qui favorise la "masse":

La masse humaine, qu'est-ce autre chose, dira-t-il, que la mer apparemment immobile mais prête à subir tous les souffles, les guettant même de tous les coins de l'horizon ${ }^{8}$.

Formé à l'école de De Maistre et de Veuillot, pouvait-il s'exprimer autrement ? Ainsi, le suffrage universel était encore pour lui en 1941 un obstacle, au même titre que la "dictature financière", à toute réforme politique ${ }^{9}$.

Le nationalisme était donc très conscient des valeurs politiques qu'il refusait: on était contre le libéralisme économique, et par le fait même contre le libéralisme politique, contre, il va de soi, le socialisme et le communisme qu'on percevait comme une émanation, une suite logique du libéralisme.

7 Ibid., 35.

8 Ibid., 22.

9 Paroles à des Etudiants, 46. 
Cependant, lorsqu'il s'est agi d'offrir des solutions de rechange, le nationalisme s'est montré particulièrement avare de développements et même d'hypothèses. On savait mieux ce qu'on refusait que ce qu'on voulait. Comme nous l'avons mentionné tout à l'heure, le passé ne pouvait plus tenir lieu de refuge. La seule forme proprement française que le Canada ait connue ne pouvait être appliquée au Québec.

Esdras Minville entretiendra une certaine nostalgie pour la monarchie française qui, par sa nature autoritaire et hiérarchisée, convenait, croyait-il, à la formation religieuse et sociale du milieu. Avec l'avènement du parlementarisme anglais qui nous était étranger, nous aurions connu alors l'esprit de parti "que le suffrage généralisé a répandu jusqu'aux couches inférieures de la société". ${ }^{10}$ En France, les mouvements de tradition conservatrice ont tôt fait de reconstituer un modèle déjà connu dans le passé. Ainsi pour les maurrassiens, le nationalisme intégral impliquait nécessairement la monarchie. Cette pièce constituait d'ailleurs la pièce maîtresse de l'ensemble.

Mais, au Québec, on n'a jamais même posé (que nous sachions), comme hypothèse, la possibilité d'instituer une monarchie. Or c'était, en politique, la seule valeur de référence globale. Devant cette absence, ce vide de tradition politique proprement québecoise, proprement nationale au niveau le plus élevé, cette droite conservatrice qui trouvait sa définition et sa raison d'être dans le passé, se voyait démunie en face du politique.

On a échafaudé, bien sûr, des paliers intermédiaires qui devaient servir à sous-tendre l'ensemble. Par le jeu des élites, on s'est proposé de restructurer le social. Il est à remarquer combien on s'est gardé d'en assurer un prolongement politique. Par moment, on a vu exalter le rôle du chef.

Le chanoine Groulx a brossé à de multiples occasions le portrait du leader charismatique auquel se fond le collectif:

10 "La Bourgeoisie et l'économique", in E. Minville, L'avenir de notre bourgeoisie, 41 . 
Le peuple sait que jadis Papineau fut un chef. Sait-il qu'il fut notre premier chef national, le premier qui ait été vraiment la voix de notre peuple, l'incarnation puissante de l'idéal, de la volonté de vivre des siens, le chef magnétique qui, pour la première fois, nous donna le sens d'une collectivité ... 11

Vague attente d'un Messie, d'un sauveur par le truchement des nouvelles élites nationales. Ainsi, Groulx réclamera un Mussolini, un Salazar, un Valera ...

Plusieurs textes révèlent la sympathie des nationalistes pour les gouvernements du Portugal, de l'Espagne, de l'Italie... en particulier. ${ }^{12}$ L'Action nationale tentera à quelques moments de présenter des portraits de chefs québecois: Duplessis y aura droit en 1933, de même plus tard, le docteur Philippe Hamel. Au fond, on s'en tiendra à l'exaltation de la nation, de l'élite qui l'oriente et finalement du chef de laquelle il est issu, en sachant très bien que l'action auprès de l'ensemble de la population n'a pas grand poids. Les nationalistes de la trempe du chanoine Groulx ne se faisaient pas tellement d'illusions quant à leur influence sur le grand public. Ils tentaient plutôt de former une nouvelle génération en rupture avec l'aboulie des précédentes. Il faut bien reconnaître que le nationalisme de cette époque n'a pas pensé se servir du politique dans son entier comme dynamique sociale. Le rôle dévolu à l'État est demeuré vague pour ne pas dire absent. Le "politique d'abord" de Maurras n'avait pas tellement de sens au Québec où la mystique avait un caractère d'antériorité dans la chronologie des réalisations nationales.

La nation, entité à consonance éminemment politique, était finalement défendue par une idéologie qui à l'époque se voulait apolitique sinon antipolitique.

11 L. Groulx, Directives (Ed. du Zodiaque, Montréal, 1937), 164.

12 "Et vous, jeunesse ... faites que, par tous vos labeurs et par toutes vos prières, nous arrive ce qui, pour tout peuple trop affaissé, est une indispensable condition de ressaisie, faites que nous arrive ce qui est arrivé au Portugal, à l'Espagne, à l'Irlande, à la Pologne, à l'Italie, même à la Turquie: un chef, un entraîneur, un incitateur d'enthousiasme et de volonté...". Lionel Groulx, "La bourgeoisie et le national", in L'avenir de notre bourgeoisie, 125. 
Le nationalisme a conçu l'économique et le politique selon la même approche. Il a cru à l'unanimité, par opposition à la notion de conflit que présentent nécessairement l'économique et le politique. On espérait faire dériver ces deux systèmes $\mathrm{du}$ social d'une "doctrine" à laquelle ils auraient obéi et assujettir l'économique et le politique aux lois du national et non l'inverse. Le nationalisme s'est défini d'ailleurs en termes religieux. Il fallait en premier lieu avoir la foi en la nation et croire à la mystique - le terme a déjà été employé qu'impliquait l'appartenance à la nation. Cette nouvelle communion des saints faite d'harmonie, d'intégration de tous les éléments ne pouvait être que gênée par des concepts de conflit. Le choix s'est fait de façon brutale: ou bien le conflit, donc la division et l'anarchie, ou bien l'unité, la cohésion, c'est-à-dire le "national". Il ne faut donc pas s'étonner de la tournure plutôt manichéenne du mouvement. ${ }^{13}$

La vision du monde extérieur demeurait, il va de soi, conforme aux exigences de la logique interne du système. L'unité nationale imposait une vue très exclusive de l' "autre" ou des "autres". Peu d'éléments extérieurs demeuraient compatibles avec l'univers passablement fermé qu'on s'était constitué. Les "métèques" dans la cité - pour reprendre un terme cher à la droite française - étaient légion. Ce nationalisme s'est défini en opposition au matérialisme américain et anglo-saxon en général, si bien que tout produit "made in U.S.A." devait être écarté du national. Les Israélites établis à Montréal présentaient un danger encore plus immédiat puisqu'ils menaçaient notre existence économique déjà fort ébranlée. Vis-à-vis de la France, une distinction s'imposait: il y avait la bonne et la mauvaise. On a rapidement trié sur le volet les bons Français: c'était souvent

${ }^{13}$ Dans une conférence faite au Collège Sainte-Marie, le 2 novembre 1937, le chanoine Groulx disait: “Je ne fais point de politique. Je n'en ferai jamais. Je n'appartiendrai jamais à aucun parti politique, quel qu'il soit. Je laisse ce marécage, ou cette fosse, à ceux qui, par goût, par nécessité ou par devoir, veulent bien $\mathrm{y}$ descendre. J'appartiens à ma conscience, à ma foi, à l'Eglise. Cela me suffit. Mais historien, l'histoire politique m'appartient comme toute l'histoire. Appuyé sur le passé, j'ai le droit d'indiquer à mes compatriotes la ligne de leur avenir." On trouve cette citation dans R. Rumilly, Henri Bourassa (Ed. Chanteclerc, 1953), 760. 
ceux qui perpétuaient la tradition d'une fidélité aux valeurs de l'ancien régime.

Finalement, envers l'interlocuteur le plus immédiat, le Canada d'extraction britannique et ses représentants à Ottawa, les positions, on le sait, ont été variables. Tantôt méfiance, tantôt refus, c'est ainsi que ce nationalisme a oscillé entre l'autonomisme et l'indépendantisme plus ou moins avoué.

Cependant, malgré le monolithisme de cette doctrine, telle qu'elle s'offrait au début des années trente et que certains comme le chanoine Groulx ont maintenue, est apparue vers les années '33 et suivantes, une multitude de rejetons issus de ce nationalisme original. Par-delà ces conceptions rigides, de nouvelles synthèses se sont réalisées avec des valeurs puisées aux sources les plus diverses partant du personnalisme de Mounier à certaines formes plus ou moins larvées de fascisme.

On doit noter au passage l'importance de l'encyclique Quadragesimo Anno; car on retrouvera dans nos idéologies au cours des années trente et quarante les mêmes ambiguïtés dont elle était porteuse. Ni capitalistes ni socialistes, les solutions proposées à cette époque étaient susceptibles de maintenir une confusion de laquelle on mit beaucoup de temps à se dégager. Une troisième voie n'était pas encore clairement tracée. A travers les multiples péripéties de la crise, de la guerre (la conscription), du Bloc populaire, deux tendances émergeront, l'une préoccupée du social - Laurendeau en sera le parangon - l'autre, conservatrice, en prolongement du type idéal des années trente et plutôt attachée à la dimension "protection" du national. A travers les seize années du gouvernement de l'Union Nationale, le social retiendra de plus en plus les uns alors que les autres y verront une défense mieux assurée du national par le biais d'une autonomie obtenue parfois à grands coûts sociaux.

Les années soixante révéleront une diversité encore plus grande des formes adoptées par le nationalisme. Parti Pris se posera à l'antithèse de la pensée sociale, économique et politique développée par le nationalisme de Groulx. Cependant, il serait intéressant de relever les ressemblances et continuités. 
Si le nationalisme officiel, comme on vient de le montrer, n'a professé que du mépris envers les partis et la politique, on ne peut pas dire la même chose des partis envers le nationalisme. Ceux-ci n'ont pu ignorer les courants nationalistes et les idées qui s'y sont exprimées. D'ailleurs, certains observateurs de la politique au Québec ont même proposé des interprétations des victoires et des défaites de nos partis qui ne tiennent compte ou presque, que de leurs positions nationalistes ou anti-nationalistes. ${ }^{14}$

C'est ainsi que les libéraux fédéraux auraient toujours dominé au Québec, depuis 1896, à l'exception de 1958, parce que des deux grands partis ce serait celui qui s'est toujours montré le plus favorable aux Canadiens français. De même, l'Union Nationale aurait supplanté les libéraux, en 1936, parce qu'elle serait alors apparue plus nationaliste qu'eux; et l'intermède libéral de 1960 à 1966 s'expliquerait également par la conversion de ce parti à un certain nationalisme, après vingt années passées à endosser les politiques centralisatrices des libéraux d'Ottawa.

Ces interprétations contiennent une part de vérité, mais elles ne suffisent pas à expliquer certains phénomènes comme la victoire des conservateurs au Québec en 1958, les succès créditistes depuis 1962, ou encore la victoire des libéraux provinciaux en 1960, puis leur défaite en 1966. Ce n'est qu'en situant les positions nationalistes des partis québecois dans un ensemble plus vaste qu'on pourra les caractériser et donner une juste évaluation de l'importance qu'elles ont eue pour eux, et plus particulièrement pour les publics électoraux qui déterminent finalement le sort des partis.

Afin d'éclairer notre lanterne, nous allons distinguer les positions prises par les partis du Québec, que ce soit au cours des campagnes électorales ou dans l'action gouvernementale, selon trois critères, ou mieux trois traits pertinents. Ces positions peuvent, d'abord, concerner la société québecoise elle-même, ou

14 On pense en particulier à Herbert F. Quinn dans son livre, The Union Nationale (Toronto, University of Toronto Press, 1963). 
bien elles peuvent concerner les relations de cette société ou de certains groupes de cette société avec l'extérieur, c'est-à-dire le gouvernement fédéral, les pays sous-développés, etc. Nous parlerons alors de positions internes ou externes que peuvent prendre les partis politiques. On doit noter tout de suite que les positions nationalistes sont toujours, par définition, des positions externes avant tout, au sens où nous venons de les définir. Dans la mesure, en effet, où le nationalisme privilégie la nation dans son ensemble, plutôt que les différents groupes ou les différentes classes qui la composent, il subordonne nécessairement l'interne à l'externe. Ce n'est pas dire qu'on doit se refuser à parler de positions nationalistes internes, mais comme nous venons de l'indiquer, ces positions, quand elles existent, sont toujours subordonnées à d'autres positions qui sont, elles, externes.

Deuxièmement, les positions prises par les partis, qu'elles soient internes ou externes, peuvent être divisées, de façon très générale, en positions socio-économiques et en positions politiques. Nous entendons par positions socio-économiques tout ce qui concerne une société donnée ou encore les relations entre sociétés, à l'exclusion de l'appareil et des mécanismes gouvernementaux qui régissent cette société, ou qui contribuent à aménager la coopération ou le conflit entre sociétés distinctes. Les positions politiques sont celles qui renvoient à cet appareil et à ces mécanismes. On aura ainsi des positions internes d'ordre socioéconomique (la réforme de l'éducation, par exemple), et des positions internes d'ordre politique (la réforme de la loi électorale, par exemple). On aura également des positions externes d'ordre socio-économique (la nationalisation de l'électricité, par exemple, dans la mesure où elle vise des intérêts étrangers), et des positions externes d'ordre politique (l'indépendance politique du Québec, par exemple).

Le nationalisme qui, encore une fois, privilégie les positions externes, peut évidemment être de nature socio-économique ou bien de nature politique. Les deux derniers exemples que nous avons donnés illustrent assez bien cette différence. Bien sûr, la nationalisation de l'électricité suppose une intervention politique, 
c'est-à-dire la mise en branle de certains mécanismes gouvernementaux, mais elle ne modifie pas, directement tout au moins, ces mécanismes et l'appareil gouvernemental qui les anime. Elle modifie plutôt les rapports de force entre des groupes qui sont de nature socio-économiques, même si elle augmente également la puissance de l'appareil gouvernemental. On comprendra que cette distinction, comme toute distinction d'ordre analytique, ne partage pas la réalité en deux sous-ensembles parfaitement imperméables l'un à l'autre. Elle nous semble, toutefois, suffisamment précise pour être utilisée avec profit dans les développements qui vont suivre.

Enfin, nous voudrions proposer un troisième trait pertinent, qui est peut-être plus risqué que les deux précédents, mais qui nous semble nécessaire pour caractériser suffisamment les positions nationalistes ou autres des partis politiques. Ce trait renvoie plus ou moins à la distinction classique, mais somme toute assez obscure, entre la gauche et la droite, qu'elle précise à partir d'un critère qui a le mérite d'être relativement simple. Nous dirions que les positions des partis peuvent être égalisatrices ou nonégalisatrices des chances ou encore des ressources de tout ordre dont disposent les collectivités concernées, qu'il s'agisse de relations internes à une société, de relations entre groupes de sociétés différentes ou, encore, de relations entre sociétés différentes - ou qu'on estime différentes.

Davantage qu'au sujet des autres traits distinctifs, on doit noter ici qu'une même mesure, et donc une même position prise par un parti, peut fort bien être égalisatrice à certains égards et non-égalisatrice à d'autres égards. Soit le cas de la réforme de l'éducation en milieu rural. Cette réforme peut être considérée comme égalisatrice pour les ruraux dans la mesure où elle diminue l'écart entre les chances respectives d'un jeune urbain et d'un jeune rural d'atteindre un niveau de scolarité supérieur au secondaire. Mais, par les longues périodes de transport en autobus que cette égalisation des chances implique, elle peut fort bien être aussi considérée par les parents du milieu rural, comme non-égalisatrice et même inégalisatrice par rapport aux 
milieux urbains. De même, une certaine déconfessionnalisation impliquée par cette réforme, dans la mesure où elle correspond à une perte de privilèges de la part de la majorité catholique, peut apparaître aux yeux de cette majorité comme égalisatrice, mais à ses dépens. On voit donc que cette notion de position égalisatrice ou non-égalisatrice est assez complexe, et qu'elle exige d'être appliquée avec précaution. A ces conditions, elle est toutefois fort utile pour caractériser les positions nationalistes ou autres des partis.

Les positions internes ou externes, qu'elles soient d'ordre socio-économique ou d'ordre politique, peuvent donc être égalisatrices ou non-égalisatrices, ce qui nous donne au total huit types possibles de positions (voir la figure 1).

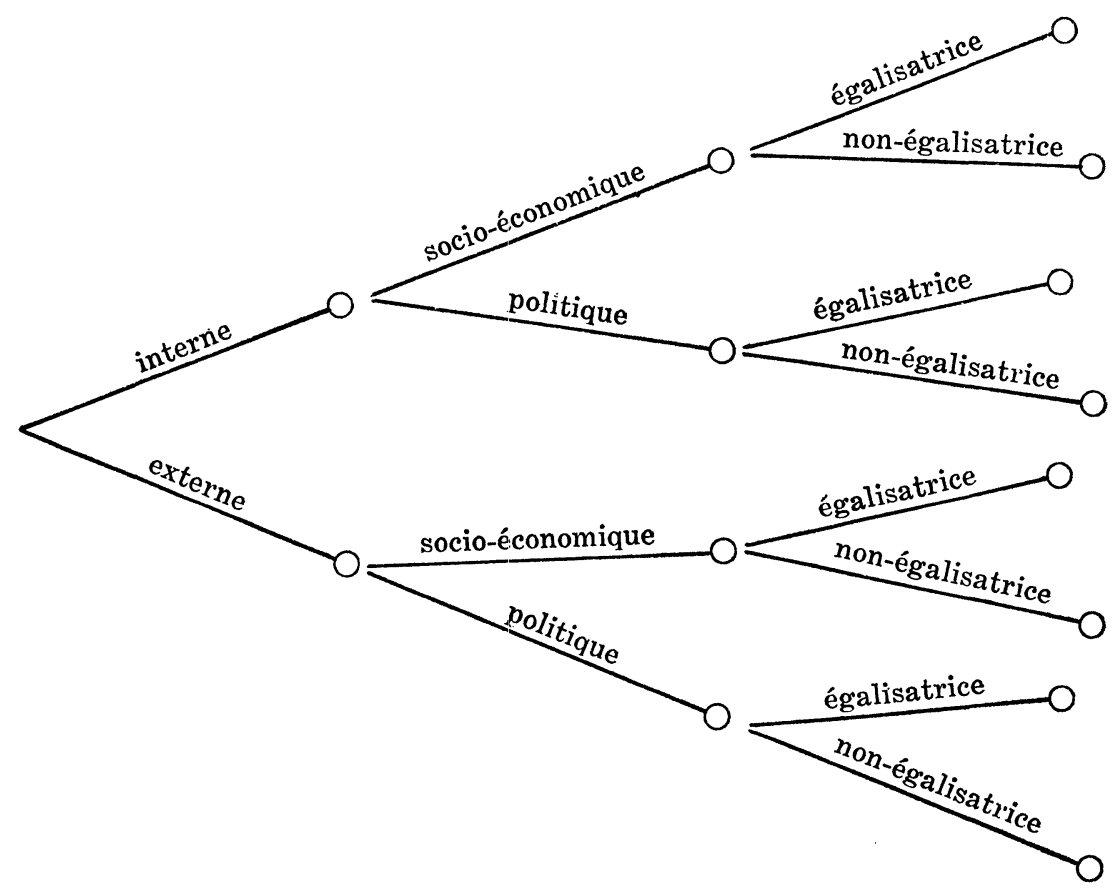

Figure 1: Huit types différents de positions qui peuvent être prises par un parti politique. 
On pourrait évidemment ajouter d'autres traits distinctifs de nature dichotomique, dont chacun multiplierait par deux le nombre déjà atteint de types possibles. Ce serait toutefois introduire une trop grande complication, qui n'est d'ailleurs pas exigée par les fins de cette analyse.

A l'aide de ces distinctions et des types qu'elles définissent, nous allons maintenant tenter de donner une interprétation moins simpliste que celles que nous avons évoquées plus haut de l'utilisation que les partis fédéraux et provinciaux du Québec ont faite du nationalisme depuis 1935. Plutôt que de faire une revue historique de cette utilisation, nous allons établir un certain nombre de propositions que nous tenterons de vérifier au moyen des données historiques dont nous disposons.

$1^{\circ}$ Il nous apparaît d'abord, comme nous l'avons d'ailleurs laissé entendre plus haut, que la plupart des grands déplacements électoraux qui se sont produits depuis 1935 ne s'expliquent pas principalement par les positions nationalistes des partis. Nous incluons parmi les grands déplacements électoraux, ceux qui se sont produits aux élections provinciales de 1936 et de 1939, ainsi qu'aux élections fédérales de 1958 et $1962 .{ }^{15}$

Les positions nationalistes de l'Union Nationale ne sont pas indifférentes à sa victoire de 1936, mais ce sont davantage ses positions internes socio-économiques et surtout politiques qui semblent expliquer son triomphe. Notons d'abord que l'Union Nationale ne jouit d'aucun avantage sur les libéraux en ce qui concerne les positions externes politiques: le parti libéral apparaît encore à ce moment comme un parti autonomiste, donc égalisateur des pouvoirs entre Ottawa et Québec, et d'ailleurs la campagne de 1936, comme celle de 1935, porte assez peu sur ce thème. Par contre, l'Union Nationale, davantage en 1935 qu'en 1936, se veut égalisatrice dans l'ordre socio-économique externe. Ses positions contre les trusts étrangers s'inspirent évidemment du nationalisme de l'époque. Mais à y regarder de plus près, un

15 Ce sont les seuls cas où les déplacements qui affectent les deux principaux partis sont au total d'au moins $20 \%$ par rapport à l'élection précédente. 
Duplessis apparaît vite plus timidement égalisateur qu'un T.-D. Bouchard par exemple, ${ }^{16}$ si bien que la différence n'est pas si grande qu'on veut bien le faire croire entre les deux partis sur ce point. Il en va d'ailleurs un peu de même des positions internes socio-économiques, où l'Union Nationale apparaît toutefois plus nettement égalisatrice que les libéraux. Mais c'est peut-être surtout sur des questions internes d'ordre politique que se joue vraiment l'élection. C'est l'image d'un parti non-égalisateur, qui utilise à son profit les mécanismes gouvernementaux, le parti de la loi Dillon et des scandales révélés au fameux comité des comptes publics, qui amène sans doute plusieurs électeurs à abandonner les libéraux.

Pourquoi alors le retour en force des libéraux trois ans plus tard? Ici les positions nationalistes ont certainement un poids plus grand, et c'est d'ailleurs devenu un lieu commun d'expliquer la remontée libérale par l'intervention des ministres libéraux d'Ottawa, agitant le spectre de la conscription. Autrement dit, les libéraux provinciaux n'apparaissent pas encore comme centralisateurs, donc non-égalisateurs, en politique externe et, surtout, ils apparaissent comme des alliés plus sûrs que l'Union Nationale pour épauler les libéraux d'Ottawa, contre les conservateurs dont le passé "impérialiste" et "conscriptionniste" continue de faire peur aux électeurs du Québec. Les libéraux, répète-t-on, sont le seul rempart contre la conscription. L'Union Nationale a beau s'affirmer elle aussi comme un parti autonomiste, les électeurs n'en semblent pas convaincus. Les thèmes nationalistes dominent donc cette élection, mais il ne faudrait pas négliger pour autant les thèmes internes socio-économiques et politiques. Les libéraux de Godbout capitalisent certainement sur une certaine incapacité du parti de Duplessis à réaliser dans l'ordre interne des mesures socio-économiques et politiques qui égalisent les chances des groupes sociaux. La rupture avec Duplessis des principaux leaders de l'Action Libérale Nationale

16 C'est du moins ce qui se dégage de la thèse de maîtrise de Claude Boileau, "Les Partis politiques provinciaux du Québec et le problème de la nationalisation de l'électricité" (Québec, Faculté des Sciences Sociales, 1966). 
donne d'ailleurs beaucoup de publicité à cette incapacité, qui semble avoir été surtout ressentie dans les milieux urbains, les mêmes qui avaient appuyé massivement l'Action Libérale Nationale en $1935 .^{17}$

Enfin les deux grands déplacements électoraux successifs de 1958 et de 1962 sur le plan fédéral peuvent être expliqués sans tenir compte, ou presque, des positions nationalistes des partis. Dans les deux cas, c'est surtout l'impact des positions internes, socio-économiques ou politiques, prises par les conservateurs puis par les créditistes, qui semblent avoir déclenché les deux déplacements massifs des électeurs québecois. Dans les deux cas, également, ces positions se voulaient plus égalisatrices que nonégalisatrices: les conservateurs ont insisté sur le caractère dictatorial du gouvernement libéral et les créditistes sur la déchéance des vieux partis, trop éloignés du peuple. La victoire des conservateurs au Québec, en 1958, s'explique également par le retrait de Louis Saint-Laurent, qui symbolisait pour les électeurs une certaine garantie de la présence du Québec à la direction du pays, mais ce retrait n'a eu pour effet que de neutraliser la dimension externe des positions partisanes, permettant ainsi aux positions politiques internes de faire toute la différence. De même les succès créditistes de 1962, s'ils se fondent très partiellement sur des positions socio-économiques externes non-égalisatrices comme, par exemple, le thème de l'aide qui doit être apportée aux gens de chez nous, plutôt qu'aux pays sous-développés, s'expliquent beaucoup plus par les positions internes d'ordre politique que nous avons déjà évoquées et surtout par des positions socioéconomiques également internes, et égalisatrices elles aussi.

$2^{\circ}$ Par contre dans les périodes plus stables, où l'on n'assiste pas à des déplacements électoraux massifs, les positions nationalistes des partis sont généralement plus contrastées que leurs positions internes, et par là expliquent peut-être davantage les résultats électoraux.

17 On peut tirer cette interprétation des analyses de Paul Cliche sur ce sujet. Voir son article "Les Elections provinciales dans le Québec, de 1927 à 1956", Recherches Sociographiques (juillet-décembre 1961) : 343-365. 
La période qui va de 1935 à 1957 sur le plan fédéral illustre cette proposition. Sauf en 1935 , les problèmes internes, socioéconomiques ou politiques, ne prennent pas beaucoup de relief. Aussi, les libéraux, parce que malgré tout leurs positions politiques externes, concernant les relations Québec-Ottawa, apparaissent comme plus rassurantes et plus prévisibles que celles des conservateurs, jouissent toujours au Québec d'un avantage électoral considérable. La prospérité économique n'incite d'ailleurs pas à des déplacements électoraux importants. La relative stabilité que l'on observe également de 1962 à 1968 exige une interprétation un peu plus complexe. D'abord les positions internes socio-économiques des créditistes demeurent contrastées avec celles des deux grands partis, ce qui est un fait nouveau par rapport à la période 1935-1957. Du côté des positions politiques externes, le contraste existe surtout entre les libéraux et les créditistes d'une part, et les conservateurs d'autre part. Ceux-ci, sauf en 1968, apparaissent toujours comme non-égalisateurs, alors que les créditistes attachent un peu plus d'importance à l'égalisation, et que les libéraux de Pearson perpétuent une tradition d'ouverture aux demandes du Québec. De ce point de vue, l'accession à la chefferie du parti libéral de Pierre-Elliott Trudeau apparaît comme équivoque. Canadien français, il symbolise mieux que Pearson cette garantie d'égalisation, mais par certaines positions il se montre en fait davantage non-égalisateur que ce que professent les conservateurs de Stanfield. En ces années où l'économie canadienne fait pourtant problème, les positions nationalistes d'ordre politique des partis du Québec demeurent aussi contrastées sinon plus que leurs positions internes, et ce malgré l'effort des créditistes qui proposent toujours des mesures plus égalisatrices qui ne sont prises au sérieux que par certaines classes défavorisées du Québec.

Sur le plan provincial, l'effet des positions nationalistes contrastées des libéraux et des unionistes durant la période qui va de 1944 à 1960 est bien connue. Ce contraste joue d'autant plus en faveur de l'Union Nationale que la prospérité économique neutralise l'effet des positions internes que prennent les libéraux. Ce n'est d'ailleurs pas tellement sur le plan socio-économique 
que sur le plan politique interne, que les libéraux finiront par exploiter avec succès les scandales et la corruption du gouvernement unioniste et la plus grande égalisation qu'ils proposent. Il ne faudrait toutefois pas négliger l'impact auprès des milieux agricoles de leurs positions socio-économiques plus égalisatrices. Mais il faut bien voir aussi que la présence à Ottawa d'un gouvernement conservateur neutralise en leur faveur la dimension externe d'ordre politique. L'Union Nationale, en 1960 comme en 1962, ne peut plus les identifier aussi bien qu'auparavant aux centralisateurs d'Ottawa maintenant que Diefenbaker dirige le gouvernement central. Ce qui nous amène d'ailleurs à une troisième proposition, complémentaire de la deuxième.

$3^{\circ}$ Comme on l'a souvent indiqué, les positions autonomistes des gouvernements provinciaux semblent avoir d'autant mieux réussi que le gouvernement fédéral était dirigé par un parti adverse de celui qui dirigeait le gouvernement provincial. Du moins depuis $1936 .{ }^{18}$ Il est vrai que la présence des libéraux au gouvernement fédéral semble avoir précipité la défaite de l'Union Nationale en 1939, plutôt que de lui fournir une arme pour sa réélection. Mais de 1944 à 1956, les positions autonomistes de l'Union Nationale ont certainement réussi, grâce surtout à la présence des libéraux au gouvernement d'Ottawa, et elles ont sans doute eu moins d'effets en 1960 et 1962, comme nous venons de le signaler, du fait de la présence des conservateurs au gouvernement fédéral. On est tenté d'ajouter que les libéraux provinciaux qui sont redevenus autonomistes de 1960 à 1966, se sont trouvés à leur tour défavorisés, en 1966, parce que leurs amis d'Ottawa avaient repris le pouvoir en 1963.

Mais alors, on doit se demander pourquoi les électeurs du Québec ont continué durant toute cette période, à la seule exception de 1958, d'envoyer à Ottawa une majorité de députés libéraux. Comment a-t-on pu, en particulier, s'opposer indirecte-

18 De 1896 à 1936, au contraire, Ottawa est plutôt la norme du vote provincial, comme l'ont montré J. Hamelin, J. Letarte et M. Hamelin dans leur étude, "Les Elections provinciales dans le Québec", Cahiers de Géographie de Québec (octobre 1959 - mars 1960) : 5-207. 
ment aux libéraux fédéraux à chacune des élections provinciales, de 1944 à 1956, pour leur donner au moment des élections fédérales d'écrasantes majorités. Il nous semble trop facile de parler de désintéressement, ou encore comme on l'a suggéré parfois, d'une espèce de psychologie élémentaire de l'électeur qui s'amuserait à opposer les "rouges" et les "bleus" aux deux paliers de gouvernement, votant "rouge" à Ottawa quand il a voté "bleu" à Québec, et vice versa.

Nous voudrions suggérer une explication un peu moins naïve, qui renvoie aux catégories que nous avons définies au début de notre deuxième partie et à l'application que nous en avons faite dans la discussion des trois propositions.

Si l'on peut parler de telle chose que l'électorat du Québec, il nous semble se caractériser avant tout par sa volonté d'égalisation, qui se manifeste selon la conjoncture sur l'un ou l'autre des quatre plans que nous avons distingués: socio-économique interne, politique interne, socio-économique externe, et politique externe.

Cette hypothèse générale exige quelques précisions. Notons d'abord que dans les périodes stables les partis sont plus ou moins maîtres de la définition de la situation, c'est-à-dire du ou des plans où ils formuleront leur volonté d'égalisation. Généralement d'ailleurs, le plan privilégié par le parti gouvernemental ne sera pas le même que celui que privilégiera le parti d'opposition. Dans les périodes de crise toutefois, les partis sont beaucoup moins libres de définir la situation comme ils l'entendent bien. C'est ainsi, par exemple, que l'Union Nationale a pu pendant longtemps faire oublier ses mesures non-égalisatrices en politique interne par l'exploitation qu'elle faisait du plan de la politique externe, entendez les relations Québec-Ottawa, où elle s'affirmait autonomiste, c'est-à-dire égalisatrice. Mais les morts successives de Duplessis et de Sauvé, le scandale du gaz naturel, ainsi que des présomptions d'autres scandales, ont fini par créer une situation de crise où les positions égalisatrices des libéraux en politique interne ont réussi à retenir l'attention. 
On peut ajouter que les problèmes externes, parce que plus éloignés des électeurs, permettent sans doute mieux que les problèmes internes une exploitation par les partis qui ne corresponde pas nécessairement à ce qu'ils ont fait, à ce qu'ils font, ou à ce qu'ils se proposent de faire. C'est pourquoi d'ailleurs l'exploitation nationaliste de ces problèmes externes a été tellement pratiquée par les partis fédéraux et provinciaux du Québec.

Voyons maintenant comment l'hypothèse générale que nous venons de formuler s'applique à notre période.

Nous avons montré plus haut que le résultat des deux seules élections fédérales de notre période où se produisent des déplacements importants, celle de 1958 et de 1962, s'explique assez bien par les positions égalisatrices des conservateurs, puis des créditistes. Avant 1958, il n'y a que les problèmes politiques externes (relations Ottawa-Québec) qui éveillent un quelconque intérêt au Québec, la prospérité neutralisant le reste, et sur ce plan les libéraux apparaissent toujours plus égalisateurs que les conservateurs. De 1962 à 1968, la division des votes qui s'est produite en 1962 demeure assez stable, les créditistes profitant surtout de leurs positions égalisatrices dans l'ordre interne, et les libéraux profitant surtout de leurs positions égalisatrices dans l'ordre externe.

De même, la victoire unioniste de 1936 s'explique aisément au moyen de notre hypothèse. Il en est ainsi, à bien y regarder, de la victoire libérale de 1939: l'Union Nationale n'a pas rempli ses promesses d'égalisation dans l'ordre interne, socio-économique, et les libéraux apparaissent comme une meilleure garantie d'égalisation dans l'ordre politique externe, c'està-dire quant au problème de la conscription et plus généralement de la participation à la guerre. Cette garantie ne s'étant pas révélée aussi bonne qu'on l'espérait, les libéraux de Godbout sont défaits en 1944, devant deux partis, l'Union Nationale et le Bloc Populaire qui privilégient l'égalisation dans l'ordre externe. La prospérité aidant, l'Union Nationale se maintient ensuite au pouvoir jusqu'en 1960, en exploitant des positions plus égalisatrices que celles des libéraux dans l'ordre externe. C'est parce 
qu'ils réussissent à neutraliser les problèmes externes et à convaincre suffisamment d'électeurs que leurs positions internes, socio-économiques et surtout politiques, sont plus égalisatrices que celles de l'Union Nationale, que les libéraux l'emportent en 1960 ainsi qu'en 1962. Avec le thème de la nationalisation de l'électricité, ils ajoutent à leur actif, en 1962, des positions externes socio-économiques qui apparaissent plus égalisatrices que celles de l'Union Nationale. Celle-ci toutefois reprend quelques circonscriptions grâce à des positions internes socio-économiques qui commencent d'apparaître plus égalisatrices. Ces positions seront mieux exploitées en 1966 et les positions égalisatrices externes des libéraux seront neutralisées par le retour des libéraux au gouvernement d'Ottawa ainsi que par la présence des partis indépendantistes dans l'élection provinciale.

Cette conclusion à laquelle nous arrivons demeure évidemment très générale. Elle pose sans doute plus de problèmes qu'elle en résout. C'est pourquoi on ne doit la considérer que comme un point de départ vers des analyses un peu plus précises de la place qu'ont tenue les positions nationalistes dans les programmes et les réalisations des partis du Québec.

Si notre hypothèse est exacte, il faudrait en effet pouvoir répondre aux quelques questions suivantes, en vue d'une vérification plus précise.

$1^{\circ}$ Qu'est-ce qui explique que les électeurs du Québec préfèrent aussi nettement les positions égalisatrices aux positions nonégalisatrices, qui n'ont pourtant pas que des désavantages? On sait, par exemple, que le progrès économique ne se passe pas de certaines mesures non-égalisatrices. Il faudrait sans doute chercher la réponse du côté des traits culturels dominants de la société québecoise.

$2^{\circ} \mathrm{Si}$, de façon générale, les électeurs du Québec se caractérisent par leur volonté d'égalisation, est-ce que selon les classes sociales auxquelles ils appartiennent, cette volonté n'est pas plus ou moins forte, et surtout est-ce qu'elle ne porte pas sur des plans différents? Il est probable, par exemple, que les cultivateurs 
et les ouvriers se préoccupent davantage d'égalisation interne à la société, tandis que les électeurs des classes moyennes et des classes supérieures se préoccupent davantage d'égalisation externe. C'est du moins ce qu'indiquent les quelques sondages un peu précis dont nous disposons.

$3^{\circ}$ Enfin, est-ce que les différentes positions égalisatrices ne sont pas plus ou moins contradictoires entre elles? De ce point de vue, la volonté d'égalisation externe des classes supérieures et des classes moyennes ne constitue-t-elle pas pour elles un moyen de maintenir certains de leurs privilèges socio-économiques et politiques à l'intérieur de la société québecoise? Cette dernière question, à laquelle nous ne tenterons évidemment pas de répondre ici, représente peut-être finalement la plus importante de toutes celles auxquelles mène l'examen sommaire que nous avons fait de l'utilisation du nationalisme par les partis.

ANDRÉ-J. BÉLANGER et VINCENT LEMIEUX 